\title{
Automated correction of phase errors in the Advanced Photon Source linac
}

\author{
N. S. Sereno, ${ }^{*}$ M. Borland ${ }^{\dagger}$ and R. Lill ${ }^{\ddagger}$ \\ Argonne National Laboratory, Advanced Photon Source, 9700 S. Cass Avenue, Argonne, Illinois 60439, USA
}

(Received 2 January 2008; published 8 July 2008)

\begin{abstract}
Maintaining stable phasing in a linear accelerator is crucial for maintaining optimal performance. If phasing is incorrect, the beam will in general have an energy error and increased energy spread. While an energy error can be readily detected and corrected using position readings from beam position monitors at dispersion locations, this method is not useful for correcting energy spread in a system with many possible phase errors. While energy spread can be corrected by looking at beam size at a dispersive location, this typically involves a beam-intercepting diagnostic and is not compatible with top-up operation. Uncorrected energy spread results in poor capture efficiency in downstream accelerators, such as the Advanced Photon Source (APS) particle accumulator ring or booster synchrotron. To address this issue, APS has implemented beam-to-rf phase detectors in the linac, along with software for automatic correction of phase errors. We discuss the design, implementation, and performance of these detectors, as well as their use in feedback to automatically correct linac phase errors during top-up operation.
\end{abstract}

\section{INTRODUCTION}

The Advanced Photon Source (APS), at Argonne National Laboratory is a high-brightness, third-generation synchrotron light source that operates in top-up mode $75 \%$ of the time to maintain a storage ring current of $102 \mathrm{~mA}$ to $1 \%$ tolerance. The APS operating availability routinely is greater than $98 \%$. The excellent machine performance is due to many hardware and software improvements including software automation of machine operations. This paper will describe the linac-beam phase-control system, which is one of many automated tools used at APS. This system measures and corrects the phase of the beam relative to each rf system that provides acceleration in the linac. We will cover the scheme for arranging the phase detectors in the linac to maximize their usefulness, show how the phase detectors are interfaced to the beam position monitors (BPMs) and linac rf systems, describe related electronics, and discuss the software used to maintain hands-free linacbeam phasing for APS top-up operation.

The APS linac was originally designed to provide a $400-450 \mathrm{MeV}$ positron beam to the particle accumulator ring (PAR). This beam had a relatively large energy spread and emittance which the PAR was designed to accept. Eventually, linac operation was switched over to electrons when the requirement for positrons was found to be unnecessary. The energy spread and emittance of the electron beam was much lower than the same parameters for the positron beam, so shot-to-shot stability of the electron beam parameters was much less critical for good PAR accumulation efficiency. Long-term drift of the energy

\footnotetext{
*sereno@aps.anl.gov

†borland@aps.anl.gov

†blill@aps.anl.gov
}

spread due to phase errors in the linac still required periodic manual tuning by the operators, which disrupted topup.

The phase detectors presented here are applied to the problem of long-term phase drift of the beam relative to the linac accelerating structures since the shot-to-shot jitter of the electron beam is not a limitation on PAR accumulation efficiency. For the LCLS experiment at SLAC, shot-to-shot phase control on the order of $0.1^{\circ}$ [1] is much more critical due to the requirements of SASE FEL operation. At SLAC, much effort is made to reduce shot-to-shot fluctuations (as well as long-term drift) at the low-level rf system level so that unwanted rf fluctuations are suppressed before they are imposed on the beam. At APS, energy spread increase due to shot-to-shot phase noise of $0.25^{\circ}$ is easily within the PAR energy acceptance of $0.8 \%$ [2].

\section{PHASE DETECTOR CONCEPT}

Linear accelerators consist of a sequence of accelerating structures where accelerating fields are phased such that particles gain energy from each accelerating structure. Typically, one operates at a phase that maximizes the beam energy, which usually minimizes relative energy spread. The APS linac consists of three accelerating sectors known as Linac Two, Linac Four, and Linac Five (L2, L4 and L5), as shown schematically in Fig. 1. These sectors use SLEDs [3] and consist of four 2856-MHz (S-Band) accelerating structures each for particle acceleration, giving the linac up to $450-\mathrm{MeV}$ total energy after L5. A single klystron not shown in Fig. 1 drives one of our two thermionic rf guns RG1 and RG2 [4,5], which produces a beam energy of $3 \mathrm{MeV}$ after the alpha magnets. Thermionic gun RG2 is used as the primary operational gun and RG1 is the backup. 


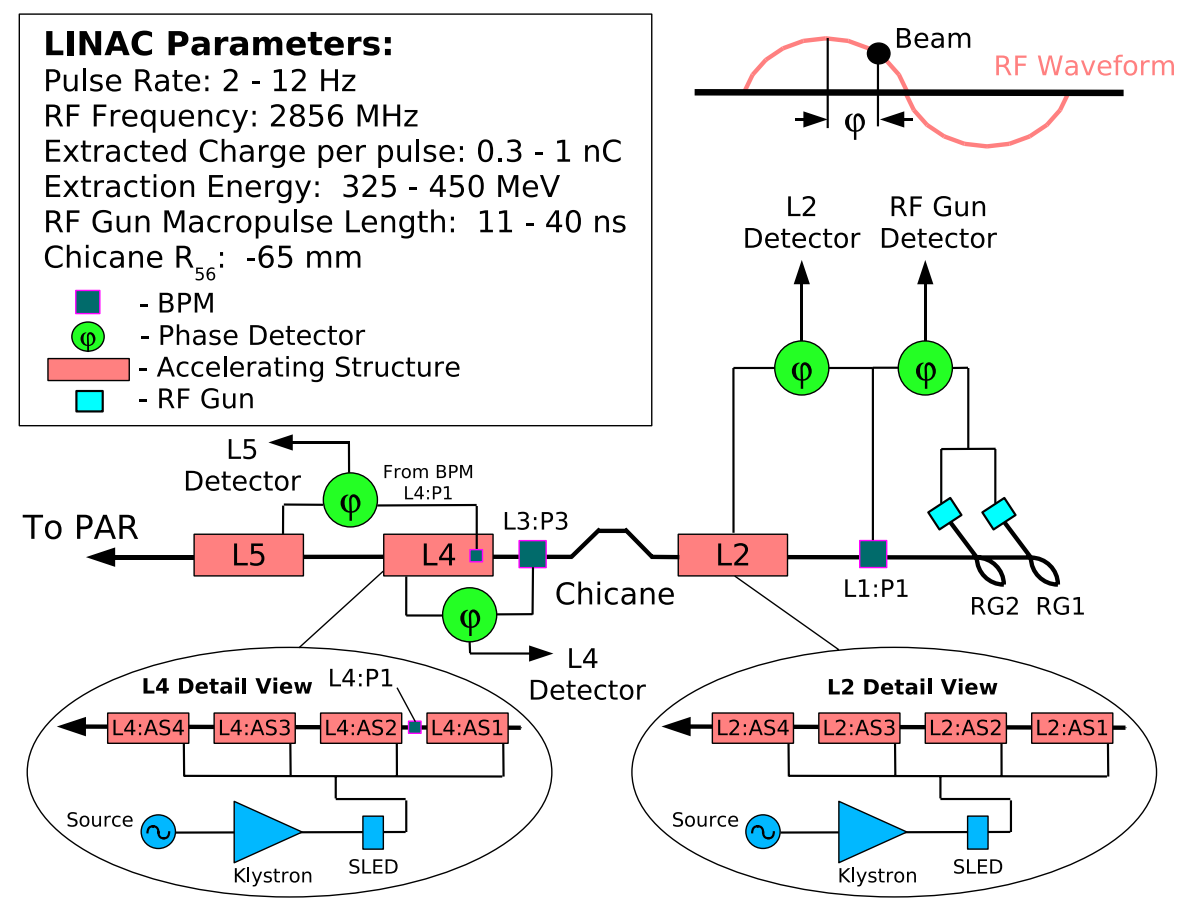

FIG. 1. (Color) Schematic of the APS linac with locations of the phase detectors labeled according to the rf system to which they attach (rf gun, L2, L4 and L5). Linac rf systems L2, L4, and L5 have a common source, klystron, and SLED configuration as shown in the bubble below systems L2 and L4. Phase detectors for systems L2, L4, and L5 are configured to take the rf signal from the ":AS1" accelerating structure. Beam position monitor L1:P1 is shared between the rf gun and L2 phase detector; BPMs L3:P3 and L4:P1 (within the L4 accelerating sector as shown) are used by the L4 and L5 phase detectors, respectively.

The chicane previously used for SASE FEL experiments is still used during operations since it provides a convenient way to maximize the energy (and minimize the energy spread) of the beam using the L2 phase. Phasing for minimum energy spread at the chicane results in a small phase offset of the beam relative to the L2 crest of $\phi_{\circ}=$ $-12^{\circ}$ where the phase sign convention is shown in Fig. 1. Operation of the beam off-crest in L2 minimizes the beam energy spread by zeroing the energy chirp of the beam emerging from the alpha magnet [4]. The chicane has $R_{56}=-65 \mathrm{~mm}$, which means that a high-momentum particle relative to the reference particle travels a shorter path. In practice, the chicane has a negligible effect on operation of the phase feedback system. Finally, the BPMs [6] used to detect the phase of the beam for the four phase detectors are called L1:P1 (shared with both RF gun and L2 phase detectors), L3:P3 used for the L4 detector, and L4:P1 used for the L5 detector.

One might think that beam-to-rf phase measurements are not necessary for the thermionic guns and the rf that drives them, since the beam has a fixed relationship with the rf phase in the gun itself. This is true for the beam upstream of the alpha magnet where the beam energy is approximately $3 \mathrm{MeV}$ from the gun. However, by comparing gun rf phase to beam phase after the alpha magnet, we will get a sensitive measurement of beam energy. This is because the alpha magnet [7] has momentum-dependent time-of-flight as illustrated in Fig. 2, given by [4]

$$
t=\frac{0.192}{\beta c} \sqrt{\frac{p}{g}},
$$

where $t$ is in seconds, $p=\beta \gamma$ is the dimensionless momentum, and $g$ is the gradient in $\mathrm{T} / \mathrm{m}$ of the alpha magnet. The derivative with respect to fractional momentum offset $\delta$ is

$$
\frac{\partial t}{\partial \delta}=0.096 \sqrt{\frac{p_{0}}{g}} \frac{p_{0}^{2}-1}{p_{0} \sqrt{p_{0}^{2}+1}},
$$

where $p_{0}$ is the reference momentum $(\delta=0)$. Typically at APS, we have $g=2.6 \mathrm{~T} / \mathrm{m}$ and $p_{0} \geq 4$. For $p_{0}=4$ (in units of $m_{e} c^{2}$ ), we have

$$
\frac{\partial t}{\partial \delta}=361 \mathrm{ps}
$$

For a $1 \%$ momentum offset, the time offset is $3.61 \mathrm{ps,} \mathrm{or}$ about $3.7^{\circ}$. The phase detector is measured to be good to about $0.5^{\circ} \mathrm{rms}$ over the long term and $0.25^{\circ} \mathrm{rms}$ shot-toshot. The shot-to-shot fluctuations are reduced by averaging when the feedback is operating. By measuring the beam phase at $\mathrm{L} 1: \mathrm{P} 1$ relative to the rf phase into the gun, we can measure the beam energy to about $0.14 \%$ rms. This is much better than we can do with the alpha magnet scraper and promises much better energy regulation than the reproducibility of the rf power allows. This is a signifi- 


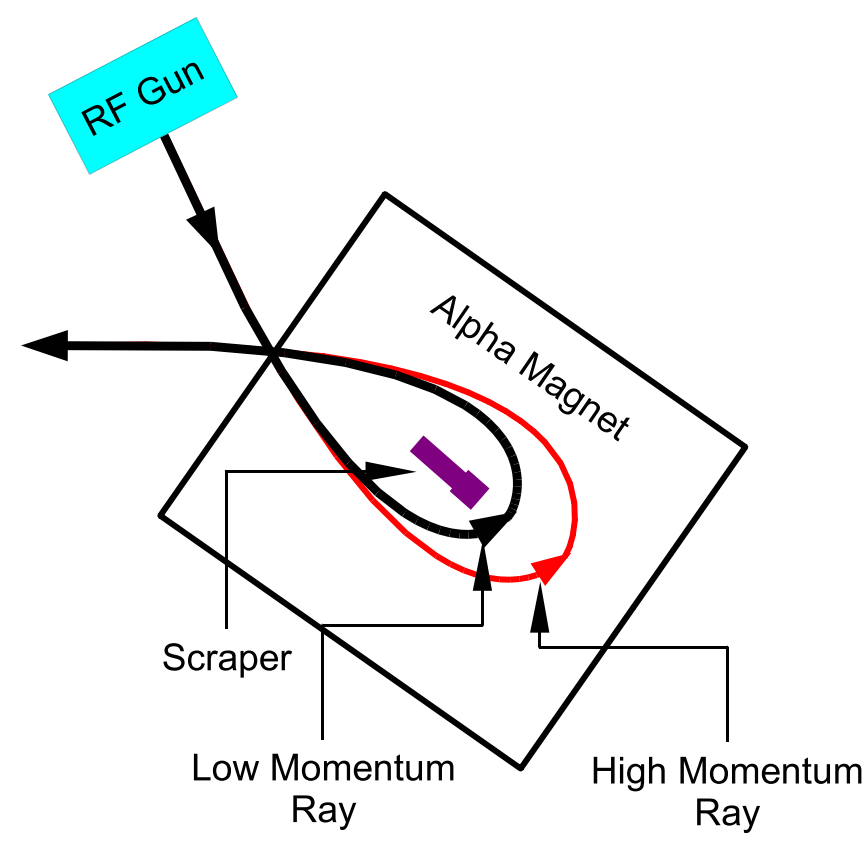

FIG. 2. (Color) Schematic of the APS linac rf gun/alpha magnet system showing that beams of different momenta take different paths through the alpha magnet.

cant benefit, given that we suspect that much of the variation in gun performance is due to variation in rf power that cannot be reliably measured directly.

Obviously, we will also want to measure beam phase relative to L2 $\mathrm{rf}$. The gradient in L2 is high enough that, if the beam is misphased sufficiently, we will not see beam at the first BPM after L2:AS1. Hence, the best beam measurement point is prior to entrance of beam into L2. Thus $\mathrm{L} 1: \mathrm{P} 1$ is the only possibility and has to be shared with the gun phase measurement.

The beam at the entrance to L4 typically has an energy of $150 \mathrm{MeV}$. If L4 is operating at high power, then it is possible to completely lose the beam before the end of L4 by misphasing. Hence, the beam measurement point should be either immediately downstream of the bunch compressor chicane, or just after L4:AS1 or L4:AS2. Since the beam is relativistic at this point, it does not matter where the phase is measured. Changes in location simply result in a constant phase delay. Therefore, we chose BPM L3:P3 immediately downstream of the chicane to measure the beam phase for the L4 phase detector.

The beam at the entrance of L5 typically has an energy of about $240 \mathrm{MeV}$. However, if L4 is down, the energy may be as low as $150 \mathrm{MeV}$. In this case, if phasing is very bad, beam may not make it to the end of L5. Hence, the L5 beam measurement point should be upstream of L5 or just after L5:AS1 or L5:AS2. There is a good reason to use a point well upstream of L5. If we used, for example, L4:P1, we could start to feed back on the phase of L5 even before beam arrived there; this means we would not have to phase L4 and L5 sequentially, which saves time. Therefore we chose BPM L4:P1 immediately downstream of accelerating structure L4:AS1 to measure the beam phase for the L5 phase detector.

\section{PHASE DETECTOR ELECTRONICS IMPLEMENTATION}

Figure 3 shows a block diagram of the phase detector electronics that determine the phase of the BPM sum signal relative to the accelerating structure rf. The phase detector receiver comprises a summing network, phase detector, and control and regulation boards. External to the receiver are power supply, data acquisition, and digital I/O boards, as well as the filtered BPM stripline and coupled accelerating structure rf signals.

The summing network block adds the horizontal and vertical stripline signals to obtain a signal that is not sensitive to beam position. Summation is accomplished by three low-loss Wilkinson 2-way power combiners. The summing block also provides gain and self-test capabilities for the system. An adjustable gain stage is also provided by the summing block, which allows one to shift the input operating range by $20 \mathrm{~dB}$ while maintaining overall system gain. In effect, the dynamic range may be extended by adjusting the summing block gain. The phase detector board S-band input signals are transformed by matching networks and then sent into the Analog Devices AD8302 log-amplifier. The AD8302 employs two closely matched wideband logarithmic amplifiers, a wideband linear multiplier/phase detector, precision $1.8-\mathrm{V}$ reference, and analog output scaling circuits. The gain and phase video output signals are then filtered and scaled to \pm 1.0 volt into $50 \Omega$. The signals are then fed into the digitizer.

Finally, the control and regulator board provides conditioned input power and housekeeping for the system. The boards are housed in an electromagnetic-interferenceshielded aluminum case. The receivers are installed in a 19-inch-wide, 4-U high card crate where up to eight receivers can be installed. The final data are sent to the EPICS control system via waveform and scalar process variables (PVs), shown in Fig. 4.

Figure 4 also shows the acquired data from the beam phase-control system receiver card. The data are acquired by the data acquisition and digital I/O card and then graphically displayed via the waveform PVs vs time. The display shows phase and amplitude waveform output of the phase detector along with slider variables (whose position are indicated by the green and red lines) that set the time range for sampling the waveform. This range is used by the detector to provide phase and amplitude around the peak of the phase detector output signal. Raw and conditioned values of voltage and phase are available, including values smoothed for use by the phase-control loop. The smoothing algorithm is implemented as a first-order digital filter. 


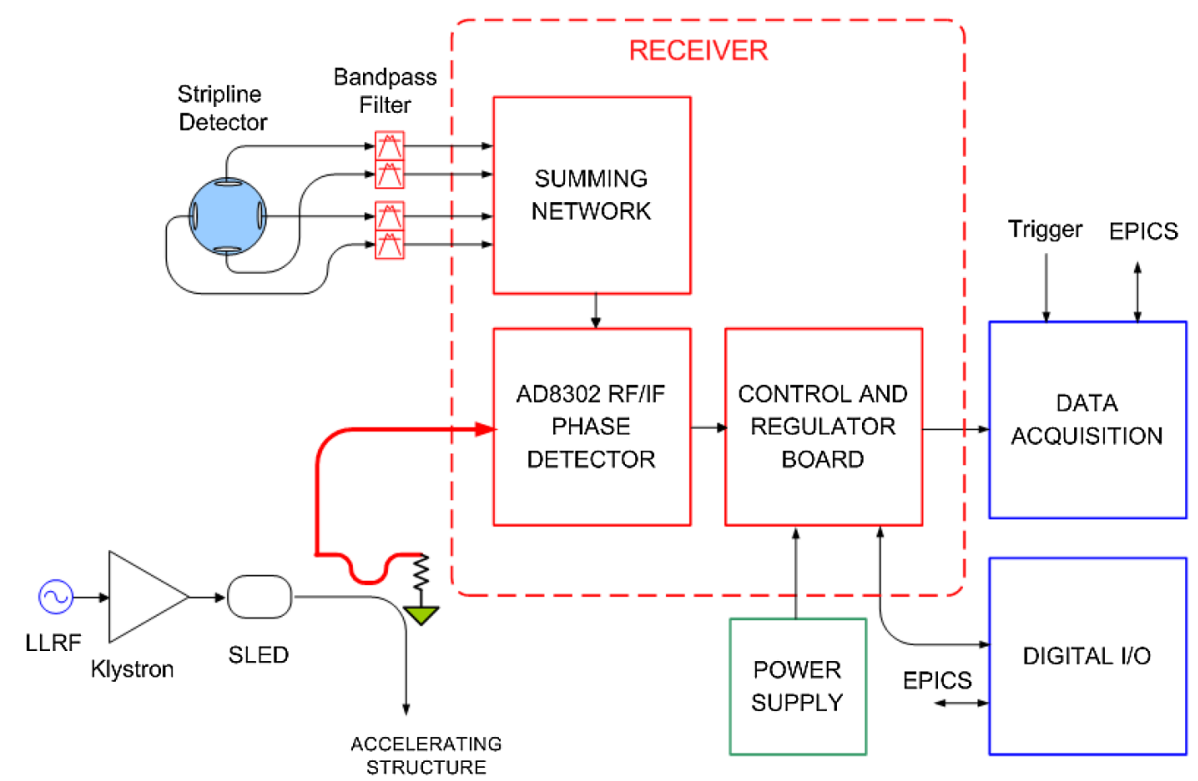

FIG. 3. (Color) Phase detector electronics block diagram.

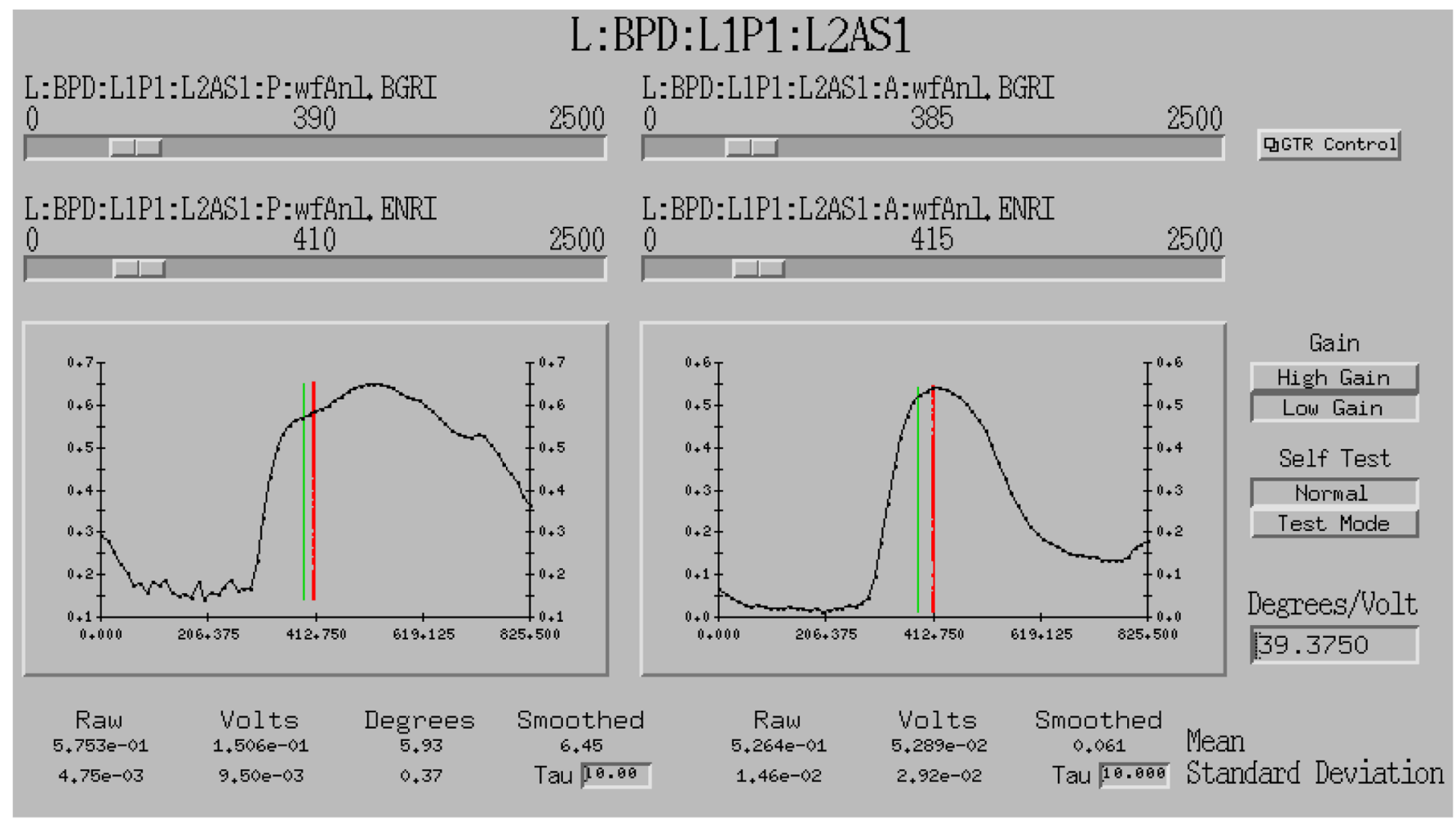

FIG. 4. (Color) EPICS waveform and scalar process variable phase detector engineering screen showing phase (left plot) and amplitude (right plot) waveforms vs time. The red and green lines specify the time range over which the phase detector acquires data and obtains the phase and amplitude. Time units are ns.

\section{PHASE DETECTOR OUTPUT VS LINAC SECTOR RF PHASE SETPOINT}

Figure 5 shows data from the L2 phase detector compared to the L2 SLED phase, which are measured relative to the low-level $\mathrm{rf}$ source, both as a function of the $\mathrm{L} 2$ phase setpoint. The data in the figure show that the phase detector linear output range is less than the full $180^{\circ}$ output of the SLED phase but is linear over approximately \pm 50 degrees of phase. The calibration of the phase detector is approximately $31.7^{\circ} /$ volt and $-35.2^{\circ} /$ volt of L2 

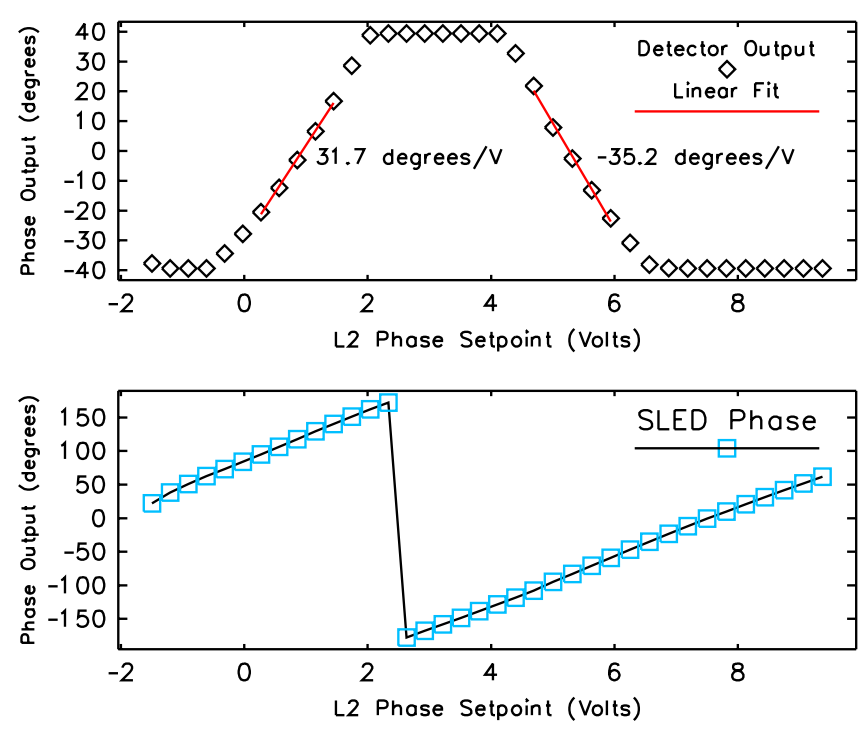

FIG. 5. (Color) L2 phase detector output compared to L2 SLED phase output vs L2 phase setpoint. L2 SLED phase is measured relative to the $\mathrm{S}$-Band source. Linear fits to the phase detector output in the nonsaturated part of the data along with the fit slope are displayed.

phase setpoint in each linear range, respectively. This is somewhat different in absolute value than the L2 SLED phase calibration factor of $36.6^{\circ} /$ volt due to the nonlinearity of the phase detector response apparent in the figure. These values are also different from the calibration factor input of $39.375^{\circ} /$ volt shown in Fig. 4 due to gain variation of the phase detector electronics. Despite this small amount of nonlinearity and gain difference, the phase detector is seen to be linear over a relatively large phase range and hence can be used in a feedback loop as long as loop operations are not attempted when the phase detector is saturated. Typically, feedback operation is performed with the phase detectors operating near $0^{\circ}$ (the middle of their range). The phase detectors are operated on the negative

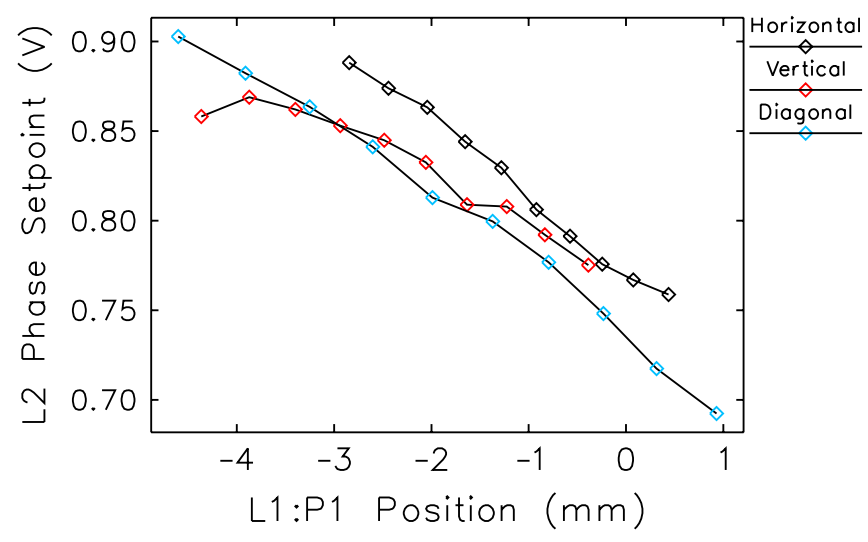

FIG. 6. (Color) Response of L2 phase detector feedback to steering at BPM L1:P1 using an upstream corrector. The results are summarized in Table I.
TABLE I. Slope of curves in Fig. 2 when the phase detector used L1:P1 to detect the beam phase. Conversion of volts to degrees was made using the absolute value of the measured negative slope shown in Fig. $2\left(-35.2^{\circ} /\right.$ volt $)$.

\begin{tabular}{lcc}
\hline \hline Plane & Slope $($ volts $/ \mathrm{mm})$ & Slope $($ degrees $/ \mathrm{mm})$ \\
\hline Horizontal & $-4.24 \times 10^{-2}$ & -1.49 \\
Vertical & $-2.33 \times 10^{-2}$ & -0.82 \\
Diagonal & $-3.80 \times 10^{-2}$ & -1.34 \\
\hline \hline
\end{tabular}

slope side of the phase response curve since this side had a slightly greater slope in absolute value.

One expects that the response of the phase detector would be insensitive to steering at the BPM since all four BPM stripline signals are summed. This is largely true; however, a small amount of phase dependence on steering at the BPM was found for the L2 phase detector, as shown in Fig. 6. The figure shows the L2 phase setpoint vs steering at the L1:P1 BPM in the horizontal, vertical, and diagonal (equal horizontal and vertical steering) planes. The L2 phase setpoint was changed by a simple phase feedback process that used the L2 phase setpoint as the actuator and the L2 phase detector as the readback to keep the measured phase at the phase detector constant.

One sees from the figure that the dependence is approximately linear. Table I lists the best (linear) fit slope for each type of steering as well as conversion of the slope to units of degrees/mm using the largest measured slope in absolute value from Fig. 5 (35.2\% /volt). Since the linac trajectory feedback processes keep the horizontal and vertical BPM readings constant to $0.3 \mathrm{~mm}$, a phase shift of between $0.25^{\circ}$ (vertical plane) and $0.45^{\circ}$ (horizontal plane) is expected due to steering reproducibility. Phase shift due to steering changes at phase detector BPMs is therefore likely the primary cause of the $0.5^{\circ}$ long-term phase variation of the feedback system.

\section{PHASE DETECTOR RESPONSE MEASUREMENTS}

The response of all four phase detectors ( $\mathrm{rf}$ gun, L2, L4, and L5) was measured as a function of the rf gun power and L2, L4, and L5 phase setpoints. The measurements were performed in two stages. In the first stage, L2, L4, and L5 phase shift actuators and detector responses were measured. In the second stage, the response of the rf gun 2, L2, L4, and L5 phase detectors was measured as a function of rf gun 2 power. The measurements were completed separately since the phase shift actuators are simple setpoint variables, whereas the rf gun 2 power is indirectly varied by means of an attenuator that is changed over a short period of time via a separate time variable. In any case, the results of the separate measurements can easily be combined into a single response matrix. The actuators were all varied about the beam's nominal phase for sectors L2, L4, and L5 and nominal rf gun 2 energy. 
The results are shown in Fig. 7. Here only a few points were used to determine the response (slope of the curves). A few points provide enough accuracy to determine the response matrix elements because, with feedback, small errors in the response matrix elements and phase detector noise are mitigated by using only a fraction (typically $50 \%$ ) of the full correction. Feedback operation uses integral averaging of phase readback errors. Integral averaging along with using a feedback gain of 50\% of full correction can correct phase errors of a few degrees on the order of 30 seconds using a correction update interval of 3 seconds.

Figure 7(a) shows the response of all four phase detectors to a change in the rf gun 2 power. Since this power changes the beam energy and hence arrival time at the entrance to L2, all four detectors register a phase shift as the response. Figures 7(a)-7(c) show only the L2, L4, and L5 phase detectors vs the L2, L4, or L5 phase setpoint actuator. In these cases, only the phase detector and phase shifter of the same sector show a response. This is because the beam is relativistic in these sectors, and therefore the arrival time at sectors downstream of the varied actuator is unchanged. In general, the arrival time at the L4 and L5 sectors will depend on the L2 phase setpoint due to the momentum-dependent path length of the chicane, which depends on the value of the $R_{56}$ matrix element for the chicane. It turns out that the effect of the chicane is to suppress the response of the L4 and L5 phase detectors relative to the rf gun and L2 phase detectors, as can be seen in Fig. 7(a). In terms of the momentum-dependent path length of the chicane $R_{56}$, the phase offset at L4 (or L5 since the beam is relativistic) due to phase offset in L2 from an rf gun power change is given by

$$
\begin{gathered}
\Delta \phi_{\mathrm{L} 4}=\Delta \phi_{\mathrm{L} 2}+\frac{\omega_{\mathrm{rf}}}{c} R_{56} \delta, \\
\delta=\frac{E-E_{\circ} \cos \phi_{\circ}}{E_{\circ} \cos \phi_{\circ}}, \\
E=E_{\circ} \cos \left(\phi_{\circ}+\Delta \phi_{\mathrm{L} 2}\right),
\end{gathered}
$$

where $\omega_{\text {rf }}=2 \pi \times 2856 \times 10^{6}$ radians/second, $c$ is the speed of light, $\Delta \phi_{\mathrm{L} 2}$, is the phase offset at L2 due to an rf gun power change, $E_{\circ} \approx 150 \mathrm{MeV}$ is the total energy gain "on crest" in L2, $E$ is the energy after a phase change $\Delta \phi_{\mathrm{L} 2}$, and $\phi_{\mathrm{o}}=-12^{\circ}$ is the beam phase relative to crest in L2 to minimize energy spread at the chicane. Equation (4) says that if the chicane were not present, phase shifts from rf gun power changes in linac sector L4 (or L5) downstream of the chicane area would equal the phase change in L2 and the gun.

As noted earlier, since $R_{56}$ for the chicane is negative, high energy particles travel on a shorter path through the chicane. Since the phase change from changing the rf gun power shown in Fig. 7 (a) is roughly $\Delta \phi_{\mathrm{L} 2}=13^{\circ}$, the phase change expected at L4 (or L5) from Eq. (4) is $\Delta \phi_{\mathrm{L} 4}-\Delta \phi_{\mathrm{L} 2} \approx 4.9^{\circ}$ or on the order of the $6^{\circ}$ to $7^{\circ}$, as seen in the figure. Qualitatively, when a positive phase change at L2 (due to rf gun power increase) increases the beam energy because the beam phase is ahead of the crest, the beam takes a shorter route through the chicane and the phase change measured after the chicane is reduced. In practice, nonlinear phase shifts due to the rf curvature in L2
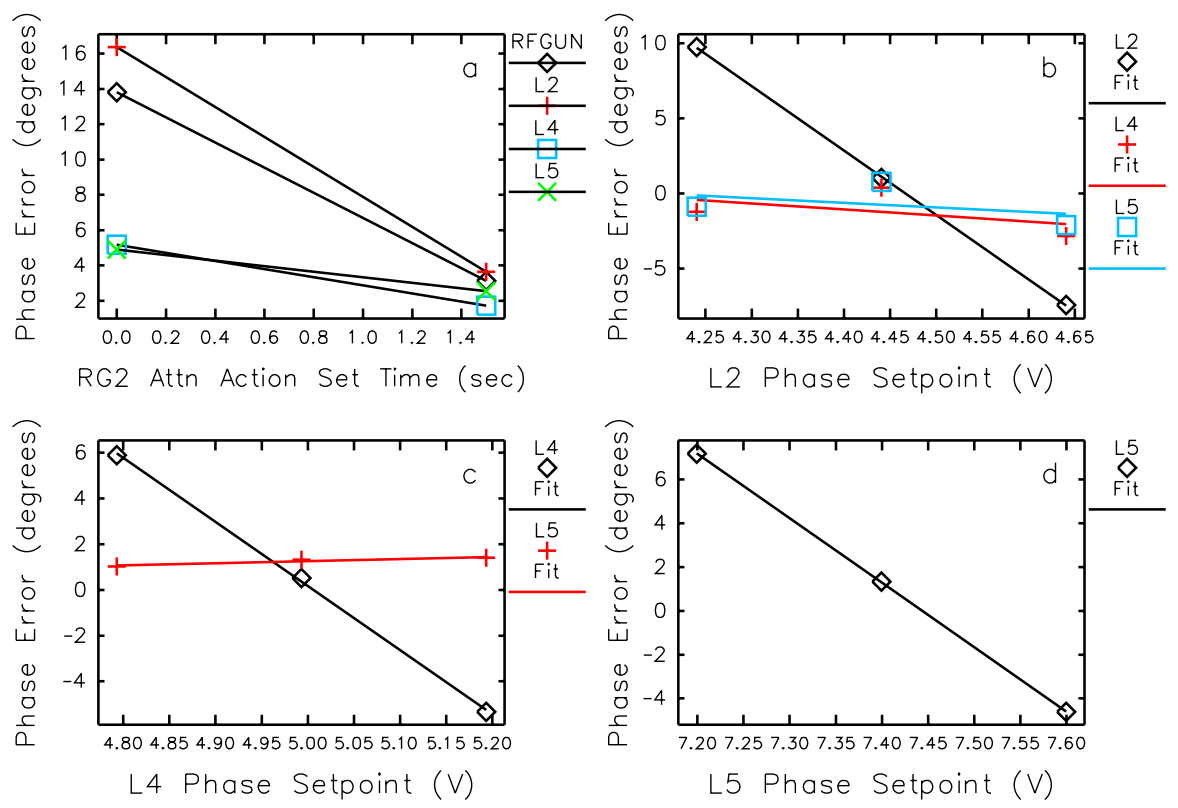

FIG. 7. (Color) Response data for all four phase detectors. The first plot (a) shows the response of all four phase detectors to a change in the rf gun 2 power. The rf gun power is changed by setting a time variable that permits an attenuator motor to move a given period of time. Plots (b), (c), and (d) show only the L2, L4, and L5 phase detectors vs the L2, L4, or L5 phase setpoint actuator. The data were all taken with the beam at its nominal phase for sectors L2, L4, and L5 and nominal rf gun 2 energy. 
do not impact action of the phase feedback since only a fraction of the full correction is applied in each feedback iteration.

\section{APS LINAC PHASE FEEDBACK IMPLEMENTATION}

The individual measured responses described in the previous section can be used to implement feedback loops in a number of ways. The simplest way is to use a single phase actuator and detector in a single feedback loop to control the beam-rf phase at a single point in the linac. Individual phase feedback was used in the initial configuration of phase control in the linac where four loops were used (rf gun, L2, L4, and L5 as shown in Fig. 1). However, if a phase error was due to an $\mathrm{rf}$ gun power fluctuation, the L2, L4, and L5 phase feedback loops would act independently to correct the phase error. Since these loops are faster than the rf gun feedback loop (which has to change the rf gun power via a stepper-motor-controlled attenuator and is slow compared to the L2, L4, and L5 loops), the L2, L4, and L5 loops would tend to correct the error then gradually move their phase actuators back to their original levels as the rf gun loop catches up. To avoid such unwarranted transients, we put all four phase detectors and actuators in a single feedback loop. In this fashion, beam-rf phase errors generated at the gun would be corrected by the gun power actuator, leaving the L2, L4, and L5 actuators undisturbed.

The matrix elements of the response matrix in this case consist of the single responses (slopes) of the four phase detector/actuator pairs shown in Fig. 7 and zeros. The response matrix has a simple form given by

$$
\begin{gathered}
\Delta \phi=\mathbf{R} \Delta \mathbf{A}, \\
\mathbf{R}=\left(\begin{array}{cccc}
R_{11} & 0 & 0 & 0 \\
R_{12} & R_{22} & 0 & 0 \\
R_{13} & 0 & R_{33} & 0 \\
R_{14} & 0 & 0 & R_{44}
\end{array}\right), \\
\Delta \phi=\left(\begin{array}{c}
\Delta \phi_{\mathrm{RFG}} \\
\Delta \phi_{\mathrm{L} 2} \\
\Delta \phi_{\mathrm{L} 4} \\
\Delta \phi_{\mathrm{L} 5}
\end{array}\right), \\
\Delta \mathbf{A}=\left(\begin{array}{c}
\Delta P_{\mathrm{RFG}} \\
\Delta V_{\mathrm{L} 2} \\
\Delta V_{\mathrm{L} 4} \\
\Delta V_{\mathrm{L} 5}
\end{array}\right),
\end{gathered}
$$

where the phase detector phase change is labeled according to the rf structure to which it is connected. The phase actuator change is a voltage in the case of rf structures L2, L4, and L5, but in the case of the rf gun, it is a power change. One sees the matrix is lower triangular, which can be understood with reference to Fig. 7. The zeros in the upper part of the matrix are due to causality. No actuator downstream of a given phase detector can affect that phase detector. The first column is nonzero due to the fact that energy errors generated in the gun affect all phase detectors, as shown in Fig. 7. The other off-diagonal elements for L2 and L4 phase detectors are negligibly small because a phase error in one sector has no impact on the downstream sectors, given that the beam is relativistic.

The inverse response matrix is also lower triangular and is given by

$$
\mathbf{R}^{-1}=\left(\begin{array}{cccc}
R_{11}^{-1} & 0 & 0 & 0 \\
-\frac{R_{12}}{R_{11} R_{22}} & R_{22}^{-1} & 0 & 0 \\
-\frac{R_{13}}{R_{11} R_{33}} & 0 & R_{33}^{-1} & 0 \\
-\frac{R_{14}}{R_{11} R_{44}} & 0 & 0 & R_{44}^{-1}
\end{array}\right) .
$$

This matrix, when multiplied by the vector of phase errors due to an rf gun phase error will only result in a phase correction to the rf gun phase actuator (power) leaving the other phase actuators unchanged. In the case of an rf gun phase error, the actuators for L2, L4, and L5 phase actuators do not move because these actuators obviously cannot correct phase errors at the rf gun. Mathematically, this can be seen from the inverse response matrix given by Eq. (11) because the first column and diagonal terms cancel only in the case of an rf gun error for sectors L2, L4, and L5. For the case of phase errors in L2, L4, and L5, only the diagonal term is involved in the correction.

\section{APS LINAC PHASE FEEDBACK OPERATION}

Figure 8 shows an example of linac phase feedback operation. The top graph shows the recovery of the phase
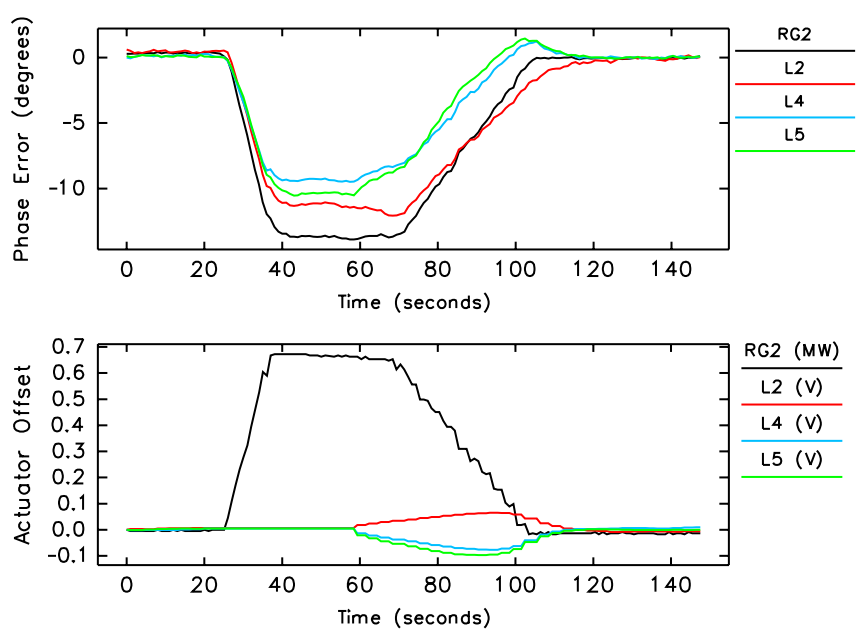

FIG. 8. (Color) Linac phase feedback operation for the case of an induced phase error due to momentarily changing the rf gun power from its nominal value. Shown is the klystron forward power, which is applied to the rf gun after being attenuated. The feedback loop is turned on approximately 60 seconds into the test. As expected, the L2, L4, and L5 phase actuators are changed only slightly. 

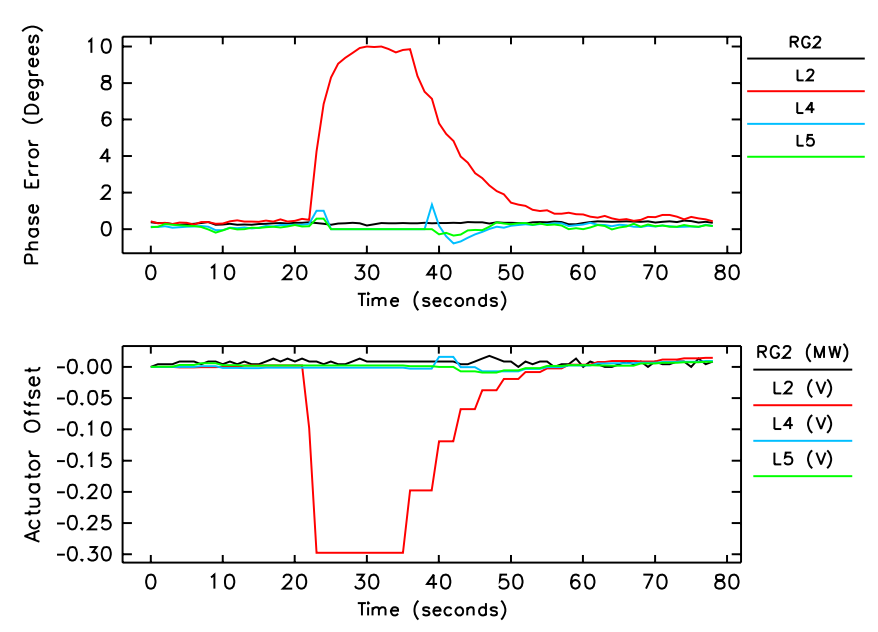

FIG. 9. (Color) Linac phase feedback operation for the case of a phase error generated by a momentary change in the L2 phase voltage actuator. The feedback loop is turned on approximately 35 seconds into the test. Operation is similar for L4 and L5 feedback loops.

actuators for an induced phase error due to momentarily changing the rf gun power from its nominal value. Only the rf gun power moves, with the L2, L4, and L5 actuators remaining nearly constant for the duration of the transient. The L2, L4, and L5 actuators do move somewhat due to noise in the system and the fact that the response matrix elements in Eq. (8) are determined by measurement and hence have some amount of error. Figure 9 shows recovery
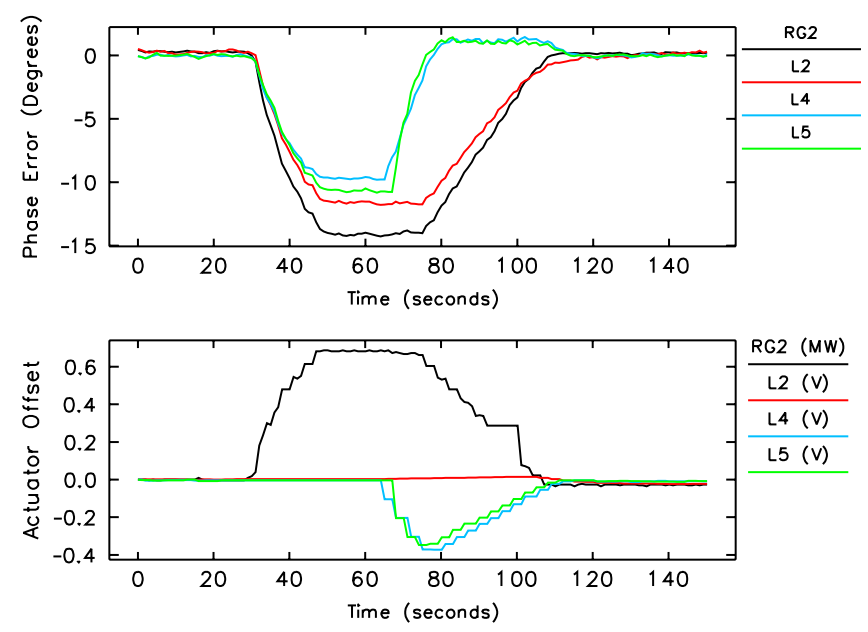

FIG. 10. (Color) Linac phase feedback operation for the case of separate feedback loops for the L4 and L5 phase detectors, with the rf gun and L2 phase detectors kept in a single feedback loop where the response matrix has the same structure as given by Eq. (8). In this test, the phase errors were induced by momentarily changing the rf gun power with the feedback loops off as in the test results shown in Fig. 8. The feedback loops were turned on at approximately 60 seconds into the test. Note the relatively large transients in phase error and actuator variables compared to those shown in Fig. 8. of the phase actuators after a phase error generated by a momentary change in the phase voltage actuator for L2. In this case, only the L2 phase actuator moves with the rf gun, and the L4 and L5 actuators remain constant. The behavior of the feedback system shown in Figs. 8 and 9 was explained in terms of the structure of the inverse response matrix in the previous section. The advantage to using a single inverse response matrix to perform the feedback is that the system (defined by all four phase detectors and phase actuators) responds by moving only the phase actuator that needs to be moved to correct the underlying phase error. This fact is particularly important for phase errors generated by power fluctuations in the rf gun since these errors are rather common during operations and machine start-up.

Figure 10 shows what happens when the single feedback loop comprising all four phase detectors and actuators is broken up into three separate loops and the rf gun power is momentarily changed with all feedback loops off. This is the same test as was shown in Fig. 8. In this case the first of the three separate feedback loops uses the rf gun and L2 phase detectors and actuators, the second uses the L4 phase detector and actuator, and the third uses the L5 phase detector and actuator. Note when the three feedback loops turn on at approximately 60 seconds two things happen. The first is that, due to hysteresis in the L1 klystron drive attenuator (which regulates power to the rf gun), the rf gun/ L2 feedback loop is slower than the individual L4 and L5
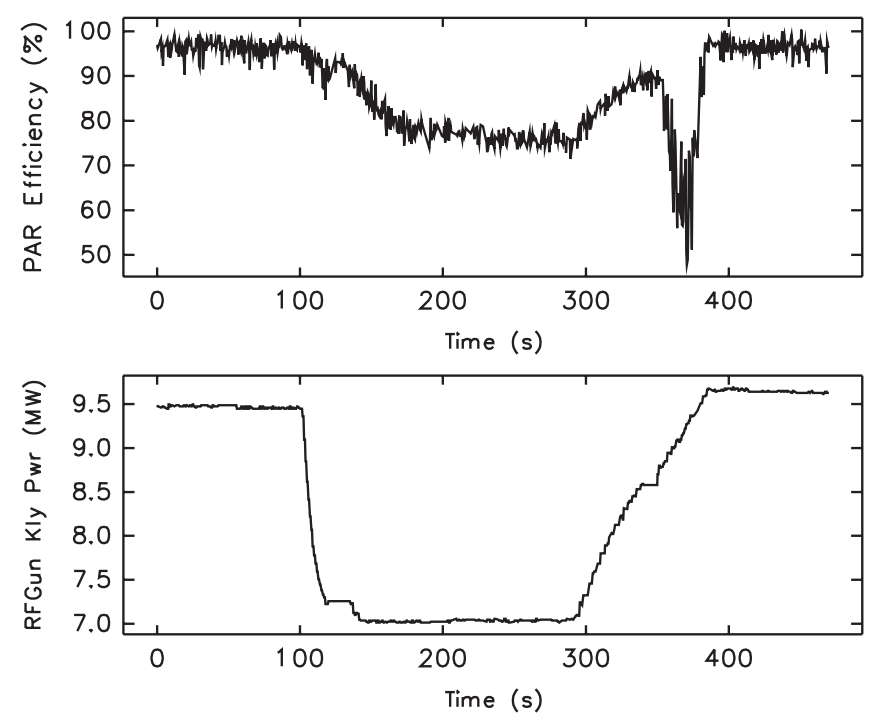

FIG. 11. (Color) Phase feedback operation impact on PAR efficiency for a large rf gun power error. At approximately $100 \mathrm{sec}-$ onds the feedback is turned off and a large 2-MW drop in rf gun klystron forward power is made as shown in the bottom plot. At 295 seconds the feedback is turned back on showing how both the rf gun klystron power recovers as well as the PAR efficiency. The large PAR efficiency glitch is due to the energy feedback overcorrecting the beam centroid energy as the phasing through the linac is restored to nominal by the phase feedback. 
loops, as seen by the recovery of the phase detector errors. The second is that the L4 and L5 actuators end up going through a much larger transient (by about a factor of 4) for the same rf gun power change compared to the transient for a single feedback that includes all phase detectors and actuators, as shown in Fig. 8. For these reasons we have implemented a single feedback for all four phase detectors and actuators in the APS linac.

Figure 11 shows the phase feedback operation impact on PAR efficiency for a large artificially induced rf gun power error. PAR efficiency is defined as the ratio of total charge extracted from the PAR after accumulation and compression divided by total charge injected into the PAR from the linac. Typically, PAR efficiency is 95 to $100 \%$. At approximately 100 seconds the feedback is turned off and a large 2-MW drop in rf gun klystron forward power is made as shown in the bottom plot. At 295 seconds the feedback is turned back on showing how both the rf gun klystron power as well as the PAR efficiency (top plot) recovers. The large PAR efficiency glitch is due to the energy feedback overcorrecting the beam centroid energy as the phasing through the linac is restored to nominal by the phase feedback.

Figure 11 is an example of how the PAR efficiency is affected by linac beam-rf phasing for an abrupt change in rf gun power. In the past, drift of the rf gun klystron power as well as other phase drift in L2, L4, and L5 not related to the rf gun power over days would result in reduced PAR efficiency due to increased energy spread. As mentioned previously, phase feedback at L2, L4, and L5 as well as power feedback on the rf gun was used prior to implementation of the beam-rf phase system described here. Although these feedback systems worked well over short periods of time, both had limitations. In the case of the phase feedback for L2, L4, and L5, the phase reference was the linac source and not the beam. So, phase errors due to rf gun mispowering and other beam phase errors throughout the linac would eventually result in increased energy spread at the PAR and reduced PAR efficiency. The rf gun power feedback used a detector that measured the out-coupled power from the rf gun waveguide. The rf gun power feedback was limited by the rf power measurement. In addition, since linac phasing using a dipole magnet and viewscreen was the primary means of restoring phasing, operations crews did not use the original feedback systems all the time and restored rf gun power and phase setpoints to different values over time scales of days. The phase detector feedback system described here requires phasing to be done manually once (using a dipole and viewscreen), and it then holds the optimum phase throughout the linac as well as the rf gun power and beam energy over the long-term.

\section{CONCLUSION}

We have described the implementation and operation of a novel phase feedback system in the APS linac. This system is based on phase detectors that give the relative phase between linac rf and beam directly. The beam phase is obtained from BPM signals and compared with coupled rf signals from the linac accelerating structures. Judicious selection of the location of the BPMs providing the beam signals allows structures to be phased even when the beam is not transmitted through the structures. Because of the use of an alpha magnet following the rf gun, precision control of the beam energy out of the gun is achieved using a phase detector.

The phase detector output of all four phase detectors is used with a response matrix to correct phase errors generated in all four linac rf structures (rf gun, L2, L4, and L5). The advantage to using a single inverse response matrix to perform the feedback is that the system (defined by all four phase detectors and phase actuators) responds by moving only the phase actuator that needs to be moved to correct the underlying phase error. Using a single matrix for feedback also has smaller transient effects compared to running separate phase feedback loops in parallel. The APS linac has operated for the past year using this system, resulting in more stable operation of the injectors during the APS's most frequently used top-up operating mode. Presently, additional phase detectors are being commissioned for use with the APS photocathode (PC) gun system. The new phase detectors will be used in similar feedback loops to keep the laser and PC gun rf locked during experiments planned to study coherent synchrotron radiation effects in the linac chicane.

\section{ACKNOWLEDGMENTS}

Much appreciation goes to Lester Erwin, Adam Brill, and Chuck Gold for installing and maintaining the phase detector electronics. Thanks to Stan Pasky who assisted with all aspects of phase detector commissioning. Gratitude goes to Eric Norum and Mariana Varotto who designed the EPICS controls and medm display screens. Thanks to Louis Emery and Glenn Decker for many useful comments and suggestions. This work was supported by the U.S. Department of Energy, Office of Science, Office of Basic Energy Sciences, under Contract No. DE-AC0206CH11357.

[1] R. Akre et al., in Proceedings of the 2007 Particle Accelerator Conference, Albuquerque, New Mexico, 2007 (IEEE, Albuquerque, NM, 2007), pp. 2421-2423.

[2] M. Borland, in Proceedings of the Particle Accelerator Conference, Dallas, TX, 1995 (IEEE, New York, 1995), pp. 287-289.

[3] Z. D. Farkas et al., in Proceedings of the 9th International Conference on High Energy Accelerators, SLAC, Stanford, California, 1974 (SLAC, Stanford, CA, 1974), pp. 1-9.

[4] M. Borland, Report No. SLAC-R-402, 1991, Sec. 3.2.4. 
[5] J. W. Lewellen, S. Biedron, A. Lumpkin, S. V. Milton, A. Nassiri, S. Pasky, G. Travish, and M. White, XIX International Linac Conference, Chicago, Illinois, 1999, Report No. ANL-98/28, pp. 863-865.
[6] R. Lill, O. Singh, and N. Arnold, Beam Instrumentation Workshop, 2002, AIP Conf. Proc. No. 648 (AIP, New York, 2002), pp. 401-408.

[7] H. A. Enge, Rev. Sci. Instrum. 34, 4 (1963). 